\title{
Acne induced by methotrexate: a case report
}

Keywords: methotrexate, cell proliferation, acute leukemia, pathology

\section{Introduction}

Methotrexate (4-amino-10-methylfolic acid) is an antagonist of folic acid that inhibits folic acid reduction and tissue cell proliferation. It is used to treat a variety of autoimmune disorders as well as hématologic or solide cancer: at two different doses for each pathology. ${ }^{1}$ This medication like others have many side effects. However, few case of acne vulgaris have been associated with the use of this treatment so fare. We are reporting a rare case of MTX-induced acne.

\section{Case report}

A 25-years-old patient, followed in the internal medicine department for acute leukemia, undergoing chemotherapy Protocol: high-dose methotrexate: $3000 \mathrm{mg} / \mathrm{m}^{2}$, ie $5040 \mathrm{mg}$ on day 1 of the Protocol followed by Purinithol from day 2 to day 7, L asparginase day 3, GRAN from D +9 to D14, and vincristine, which presented the day after the injection of methotrexate, a slightly pruriginous rash, diffused all over the face, the trunk, the back, and even the thighs, This eruption was made of monomorphic acneiform lesions, from pustules type, and diffuses inflammatory papules, without retentional lesions, with excoriations surmounted by haemorrhagic crusts (Figures 1-3) It was the 5th cycle of the patient, and he reported the appearance of his similar lesions, since first cure, 2 days after taking this high dose of MTX, with regression of the symptomatology 10 days after. In front of this conditions, the diagnosis of acne induced by MTX was retained (the other drugs were discarded since the eruption was objectified before their administration), a therapeutic abstention was recommended, with again a disappearance of the lesions 10 days after taking MTX.

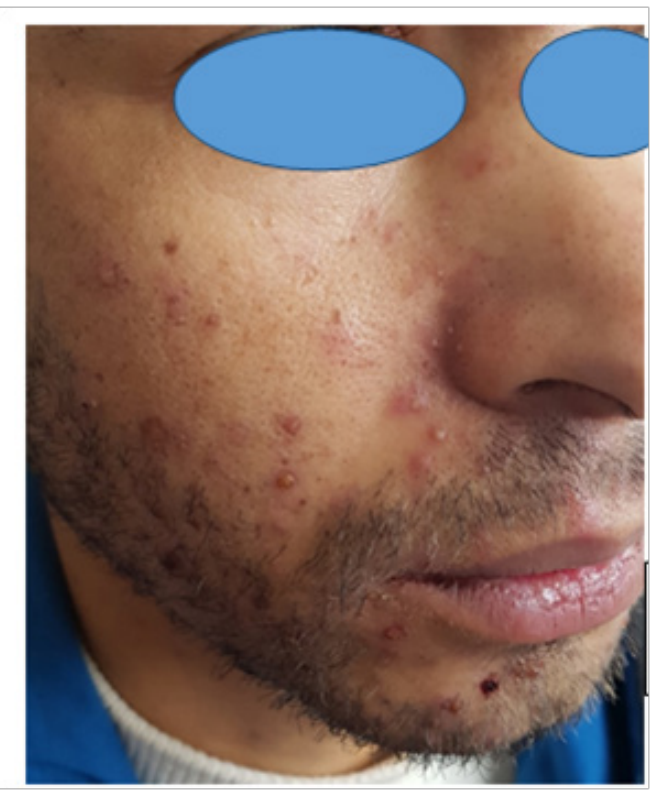

Figure I Multiple papules and pustules in the face.
Volume 3 Issue 4 - 2019

M bennani, S Benkirane, S Elloudi, Z Douhi, $\mathrm{H}$ Baybay, FZ Mernissi

Dermatology-Venerology Department CHU hassan II FES, Morocco

Correspondence: M Bennani, Dermatology-Venerology Department CHU hassan II FES, Morocco,

Email mouniaabennani24@gmail.com

Received: July 18, 2019 | Published: August 02, 2019

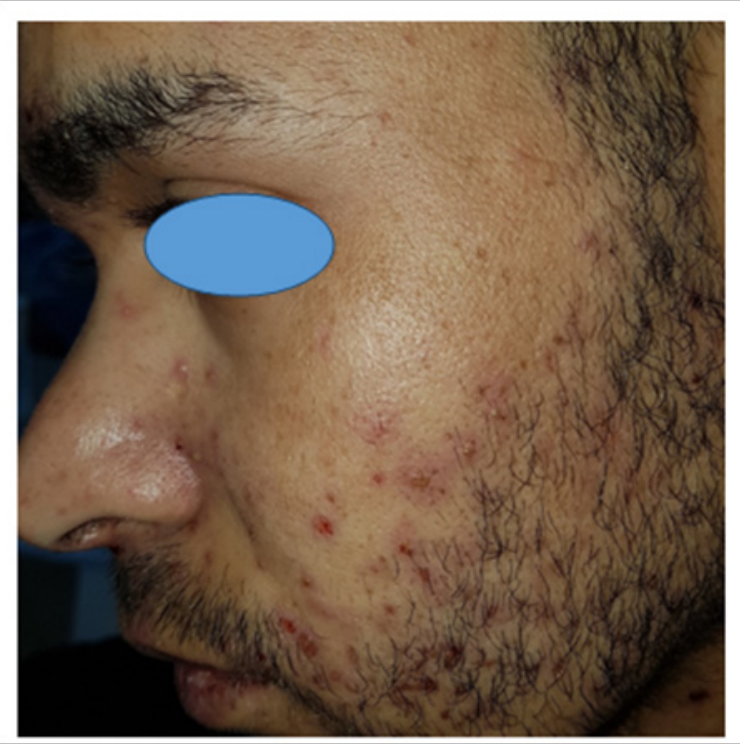

Figure 2 several excoriations surmounted of haemorrhagic crusts.

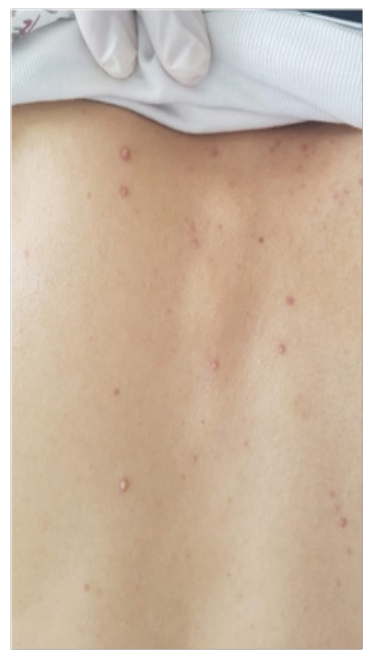

Figure 3 several papulo-pustules monomorphic at the level of the back. 


\section{Discussion}

A variety of drugs may provoke acne such as corticosteroids, cyclosporine, antipsychotics, anticonvulsants, epidermal growth factor receptor inhibitors, antidepressants, danazol, antituberculosis drugs, quinidine, azathioprine, testosterone, ${ }^{2}$ and TNF-a antagonists. ${ }^{3}$ Drugs that induced acne (DIA) are often having some specific clinical and histopathologic features. DIA is characterized by a medical history for drug intake, by a sudden onset, and at an unusual age, with a monomorphous eruption of inflammatory papules or papulopustules. The location of the acne lesions is beyond the seborrheic zone. $^{4}$

The originality of our case is the rapid onset of eruption in less than 24 hours after injection, that was explained, by the high dose taken by the patient in a single injection. The sudden onset of diffuse inflammatory acne with no predominance in the seborrheic areas at each cycle after each MTX injection, and it spontaneous disappearance 10 days later, confirmed our diagnosis of MTX-induced acne, including all necessary for this entity. ${ }^{5}$

\section{Conclusion}

We report a case of methotrexate-induced acne, rarely reported in the literature, then adding this treatment to the long list of acneinducing drugs.

\section{Acknowledgments}

None.

\section{Conflicts of interest}

Author declares that there is no conflict of interest.

\section{Funding}

None.

\section{References}

1. Rau R. Methotrexate. Z Rheumatol. 2016;75(6):599-603.

2. Momin SB, Peterson A, Del Rosso JQ. A status report on drug-associated acne and acneiform eruptions. $J$ Drugs Dermatol. 2010;9(6):627-636.

3. Emmanuelle Steels, Anne Peretz\& Pierre Vereecken. Infliximabinduced acne: A new case and review of published reports, Journal of Dermatological Treatment. 2009;20:59-60.

4. Maria Kashat BS, Katherine Caretti MD, Jessica Kado MD. EtanerceptInduced Cystic Acne WWW.CUTIS.COM VOLUME. 2014; 94:31-32.

5. Kazandjieva Jana, Tsankov N, Drug-induced acne. Clinics in Dermatology. 2016;35(2):156-162. 